\title{
Team performance indicators which differentiate between winning and losing in elite Gaelic football
}

Declan Gamble ${ }^{\mathrm{a}, \mathrm{d}}$, Jonathan Bradley ${ }^{\mathrm{b}}$, Andrew McCarren ${ }^{\mathrm{c}}$ and Niall M.

Moyna $^{\mathrm{d}}$

${ }^{a}$ Sport Northern Ireland Sports Institute, Ulster University, Newtownabbey, N.Ireland

${ }^{b}$ Centre of Performance Analysis, Institute of Technology Carlow, Carlow, Ireland

${ }^{c}$ Insight Centre for Data Analytics, Dublin City University, Dublin, Ireland

${ }^{d}$ School of Health and Human Performance, Dublin City University, Dublin, Ireland

Corresponding author:

Mr Declan Gamble

Sport Northern Ireland Sports Institute,

Ulster University, Newtownabbey,

BT37 0QB, N.Ireland

Mob: +447748861286

Email:declangamble@sportni.net 


\title{
Team performance indicators which differentiate between winning and losing in elite Gaelic football
}

\begin{abstract}
The objective of this study was to identify performance indicators which differentiated between winning and losing elite Gaelic football teams. Eighty three technical and tactical performance variables were measured in 13 teams during 26 league and championship games throughout 2014-15. Univariate analysis of full-games revealed that winners achieved a significantly higher total score, number of scores, shots, points, points from play and goals, resulting in superior shot efficiency, average attack per score, and scores per 10 possessions. Winners gained significantly more turnovers and completed significantly less unsuccessful hand passes. Winners also performed significantly less kick outs, resulting in fewer successful kick outs and successful dead ball kick passes overall. A principal component analysis, conducted on 18 variables produced 4 components, which explained $81.9 \%$ of the variance. Both logistic regression (8.00, $\left.\chi^{2}(1)=16.00, p<0.001\right)$ and discriminant analysis $\left(\Lambda=0.53, \chi^{2}(1)=13.77, p<0.001\right)$ revealed that 1 component; defensive counterattacking, significantly contributed to outcome and differentiated winners from losers with a cross-validation accuracy of $87.5 \%$. Coaches can use this information to organise their defensive system to generate opposition turnovers and also incorporate sufficient flexibility to facilitate effective transitions to exploit their own offensive counterattacking opportunities.
\end{abstract}

Keywords: Team sport, match analysis, technical variables, football 


\section{Introduction}

Gaelic football is a popular team sport played in Ireland with National football league (NFL) and All-Ireland championship (AIC) games regularly attracting significant attendances and television viewing audiences. Although Gaelic football is an amateur sport, elite inter-county players adopt a quasi-professional training regime (Beasley, 2015). Gaelic football match-play is characterised by turnovers and fast pacedtransitions, as teams counterattack and transfer the ball from their own defensive (between 0-45 $\mathrm{m}$ from goal line), midfield (between the two $45 \mathrm{~m}$ lines) or attacking (between 45-0 m from opposition goal line) zones, and try to score in the opposition's defensive zone. Furthermore, turnovers and counterattacks can contribute to enhancing the dynamic flow of the game, which is sometimes attenuated by the extreme zonal defensive strategies commonly adopted by contemporary teams (Bradley \& O’Donoghue, 2011). To understand the factors contributing to game outcome, key indicators that define aspects of performance need to be examined (Hughes \& Bartlett, 2002). Since Reilly and Collins (2008) highlighted the need for more scientific studies in Gaelic games, research has emerged documenting physical (Gamble, Spencer, McCarren, \& Moyna, 2019; Malone, Solan, \& Collins, 2017) and technical (Allister, Byrne, Nulty, \& Jordan, 2018; Bradley \& O’Donoghue, 2011; Carroll, 2013; Lynch \& Carroll, 2017; Mangan et al., 2017; McGahan et al., 2018; McGuigan, Hughes, \& Martin, 2018) performance variables and contextual factors (Rooney \& Kennedy, 2018)

Bradley and O'Donoghue (2011) reported that the majority of successful counterattacks leading to scores in AIC games during the 2007 and 2008 seasons originated in a team's defensive or midfield zone, commenced following a 
dispossession, were between $26-35 \mathrm{~s}$ in duration, involved $\geq 5$ passes and penetrated into the opposition's $21 \mathrm{~m}$ defensive zone. Only $12 \%$ of counterattacks initiated with a kick pass resulted in a score compared to $25 \%$ when a hand pass was used, the latter enabling more players to support the attacking play and for offensive players to initiate their penetrating runs (Bradley \& O'Donoghue, 2011). An analysis of AIC games in 2011 and 2012 found that 'top' teams, who reached the quarter-final stages of the AIC in 2 of the previous 3 years, recorded significantly more attacks, shots, points scored, fouls committed, and less turnovers against, when compared to 'bottom' teams, who were eliminated from the AIC prior to the quarter-finals (Carroll, 2013). The top teams also recorded a higher shot efficiency, and won a higher percentage of both their own and the opposition's kick outs. The fact that both total shots taken and attack efficiency were significantly lower when top teams played each other compared to when they played lower tier teams (Carroll, 2013) indicates that Division 1 teams deploy more effective defensive strategies.

Teams competing in the AIC semi-finals and finals during 2014-16, executed a significantly higher number of hand passes and lower number of kick passes in both defence and midfield, than teams competing in finals during the 1980s (Lynch and Carroll, 2017). Interestingly, the kick pass success rate is substantially higher among contemporary players than those who played during the 1980 s (81 vs. 55\%). This difference may be due to enhanced technical competency or to the fact that during the 1980's the kick pass was often used to transfer the ball over a long distance with less emphasis being placed on retaining possession. The significant reduction observed in forward directed passes, from the 1980's to 2014-16 (84 vs. 63\%), was associated with a significant increase in both backward and lateral passes in the same period. Further, the significant reduction in forward kick passes combined with the significant increase 
in forward hand passes during 2014-16, indicates that modern teams have adopted passing strategies that emphasise ball retention in order to create scoring opportunities that have a higher probability of success (Bradley \& O’Donoghue, 2011; Lynch \& Carroll, 2017). This is in contrast to the more direct kick passing offensive strategy used in the 1980s (Lynch \& Carroll, 2017).

The preferential use of the hand pass to transfer the ball was also demonstrated by Mangan et al., (2017) from NFL and AIC games between 2014 and 2016. Over twothirds $(70 \%)$ of passes were by hand with a retention rate of $97 \%$. This is higher than the $79 \%$ of kick passes retained. Although less than one third of all kick outs were directed within the defensive $45 \mathrm{~m}$ zone, this "short" kick out strategy resulted in a ball retention rate of $92 \%$ compared to $56 \%$ for kick outs directed beyond the $45 \mathrm{~m}$ line. The number of fouls, turnovers and tackles were aggregated into a composite performance indicator termed "defensive actions". Although fewer defensive actions occurred in attack (55), than defence (65), or midfield (55), there is potential for teams to adopt a high press (McGahan et al., 2018) to regain possession and create more scoring opportunities. When comparing Division 1 and Division 3 teams, McGahan et al. (2018) found that teams in the top division performed significantly more tackles in midfield and defensive areas and had a similar number in attack, indicating that the higher standard of play was associated with more organised defensive strategies. Although the number of successful shots from play was similar, Division 3 teams were not exposed to the same frequency of tackles when attacking and they also registered significantly more missed shots from play due in part to inferior technical proficiency (McGahan et al., 2018). 
Allister et al. (2018) used discriminatory analysis to differentiate winners from losers in AIC games between 2015 and 2017 and reported an overall classification accuracy of $71 \%$. Of the 13 performance indicators examined, fouls committed, goals scored and total attacks were associated with the highest discriminatory power. In addition to these variables, shot efficiency and black cards differentiated winners from losers in close games (winning margin $<6$ points), whereas; attack efficiency, shot efficiency, percentage opposition kick outs won, yellow, black and red cards, distinguished winners from losers in unbalanced ( $\geq 6$ points difference) games.

Performance variables differentiating winners from losers have previously been identified using univariate analysis by researchers in rugby (Watson, Durbach, Hendricks, \& Stewart, 2017), whereas multivariate models including; discriminate analysis and logistic regression, have been employed in soccer (Castellano, Casamichana, \& Lago, 2012) and Australian rules football (Robertson, Back, \& Bartlett, 2016), respectively. Although discriminate analysis is considered more powerful, logistic regression is commonly used as a robust alternative because it is not constrained by stringent assumptions (Hastie, Tibshirani, \& Friedman, 2009). Moreover, to progress methods employed previously, a principal component analysis (PCA) could be used to reduce the extensive range of performance indicators into smaller composite dimensions (Field, 2018) prior to the differentiating evaluation. Importantly, Robertson et al. (2016) expressed performance indicators in their relative form, defined as “descriptive conversion" (Ofoghi, Zeleznikow, MacMahon, \& Raab, 2013), to account for the influence of the opposition and between-match contextual factors. Therefore, the aims of this investigation were to; 1) identify relative differences in the technical and tactical performance indicators that could distinguish winning and losing Gaelic football teams, 2) use PCA to identify specific variables which could be 
combined and transformed into new performance indicators, and 3) compare the classification accuracy of logistic regression and discriminate analysis models.

\section{Methods}

\subsection{Match sample}

The technical and tactical performance indicators from winning and losing Gaelic football teams were examined during 16 inter-county Division 1 NFL and 10 AIC games over 2 seasons (2014-2015). Games were played between 1300 and $2100 \mathrm{~h}$. The match sample included 1 semi-final and 1 final from the NFL, and 2 semi-finals and 2 finals from the AIC. The other 2 AIC semi-finals involved extra time and replays and were not included. As winners and losers could not be differentiated from draws, 2 league games were excluded from the original sample $(n=26)$ for the analysis $(n=24)$. Fourteen games were associated with a small winning margin ( $\leq 5$ points), whereas the remaining 10 games involved a large win (between 6-15 points). Team ratings were determined using the Elo rating system for Gaelic football (Mangan \& Collins, 2016) and the total playing time including stoppages was used in the analysis.

\subsection{Experimental procedures}

Match footage was sourced from a combination of internal team video recordings and from external media broadcasters (BBC, Premier Sports, RTÉ, Setanta Sports, SKY and TG4). In 16 of the 24 games, 2 video sources were obtained, which enabled crosschecking of events. In some of these games and the remaining 8 games, a very small number of events (mean $\pm \mathrm{SD} ; 4 \pm 5$, range; 0 - 22) were estimated due to television replays, obscured vision and/or footage quality. Each game was transferred from a DVD to a Toshiba Satellite Pro (Tokyo, Japan) laptop computer (Intel Core i5-5200U 
CPU) operating Microsoft Windows 10 (Washington, USA). The footage was then imported using Dartfish (v8) TeamPro software (Fribourg, Switzerland).

A custom built tagging panel was used to code the games and document key performance variables. All matches were coded by the same individual (>15 years' experience analysing elite sports performance). The mean $( \pm S D)$ number of events per game was $1161 \pm 64$ (range; $1044-1308$ ), with each event involving a minimum of 3 and maximum of 11 tagging inputs. Match events included frequency counts, duration (for possession), pitch location (origin) and outcome. Following completion of the coding for each game, the events were visually inspected to detect and correct operational tagging errors. Each individual event was then examined and checked for accuracy. The sequence of events was then observed and adjusted (where necessary) to ensure that the tagging timeline captured all related events. Once the data validation was concluded, the coding events were then exported into Microsoft Excel (Microsoft, USA). Finally, the data were transformed and collated for specific match periods to facilitate statistical analysis.

\subsection{Variables and definitions}

Match characteristics included; Elo ratings, substitutions, cards received, playing time and ball in play. In addition, the 83 technical and tactical performance indicators (35 raw and 48 derived) examined in the present study (Tables 2-6) were developed following a review of Gaelic football literature (Allister et al., 2018; Bradley \& O’Donoghue, 2011; Carroll, 2013; Lynch \& Carroll, 2017; Mangan et al., 2017; McGahan et al., 2018; McGuigan et al., 2018) and were subsequently validated by an expert team of coaches and support staff (Hughes \& Bartlett, 2002; Jones, Mellalieu, \& James, 2004; O’Donoghue, 2009). Operational definitions and performance indicators 
Table 1. Operational definitions and performance indicators used during match analysis

\begin{tabular}{|c|c|}
\hline Game statistic & Description \\
\hline Playing time & Duration of playing period including ball in play time and stoppage time due to injuries or dead balls. \\
\hline Ball in play & Any period of time in which the ball is active within the boundaries of the playing field. \\
\hline Foul & Any action that is considered by the referee to be an infringement on the rules, resulting in a free kick. \\
\hline Yellow card & When a player is shown a yellow card. \\
\hline Black card & When a player is shown a black card. \\
\hline Red card & When a player is shown a red card and/or black card and not replaced. \\
\hline Substitution & When a player is replaced during the game. \\
\hline Elo rating & An objective rating of a team's current performance based on historical data. \\
\hline Player possession & $\begin{array}{l}\text { When a player controls the ball with either hand or foot. Possession persists until the player scores or fails in } \\
\text { an attempted pass or shot, or the player is dispossessed and doesn't regain possession. }\end{array}$ \\
\hline Team possession & Team possession starts with control of the ball and persists until the team scores or a player loses possession. \\
\hline Hand pass & Transfer of ball between players using the hand/fist. \\
\hline Kick pass & Transfer of ball between players using the foot. \\
\hline Pass total & Combined hand pass and kick pass. \\
\hline Dead ball & When a player releases possession from a dead ball; kick out, free kick, sideline, $45 \mathrm{~m}$ or penalty kick. \\
\hline Dead ball kick pass & When a player releases possession from a dead ball; kick out, sideline or free kick. \\
\hline Dead ball kick out & When a player releases possession from a dead ball kick out. \\
\hline Dead ball free kick pass & When possession is released from a dead ball free kick. \\
\hline Pass succes/fail & Pass successful if possession retained, unsuccessful if possession lost. \\
\hline Turnover & When possession transfers from one team to another during play (excluding kick outs). \\
\hline Tackle & $\begin{array}{l}\text { When a player attempts to dispossess an opponent who is in possession of the ball. Successful if player is } \\
\text { dispossessed and loses possession, commits a technical foul or fails to execute a pass or shot resulting in a } \\
\text { score. Unsuccessful if player retains possession or scores. Minor physical contact on an opposing player's } \\
\text { body is not counted, but contact on the ball is. }\end{array}$ \\
\hline Attack & $\begin{array}{l}\text { When a ball is passed across the opposition's } 45 \mathrm{~m} \text { line or shot attempted from outside the } 45 \mathrm{~m} \text { line. If the } \\
\text { ball re-enters the middle zone and is then passed, carried back, or shot attempted into the attacking zone, it is } \\
\text { considered the same attack. A new attack can start within the attacking zone if a turnover is gained during } \\
\text { play from a kick out, sideline kick or technical foul. Attack ceases with loss of possession. }\end{array}$ \\
\hline Shot & $\begin{array}{l}\text { An action that sends the ball directly towards the opposing teams' goal in an attempt to score a point or goal. } \\
\text { Successful if score obtained. Unsuccessful if no score obtained. }\end{array}$ \\
\hline Point & When the ball is kicked or fisted over the crossbar and between the two posts ( 1 point). \\
\hline Goal & When the ball is kicked under the crossbar and between the two posts ( 3 points). \\
\hline Total score & Combined total score from points and goals. \\
\hline Total no. of scores & Combined total number of scores from points and goals. \\
\hline Attacking efficiency & Number of shots expressed as a percentage of the total number of team attacks. \\
\hline Shot efficiency & Number of scores expressed as a percentage of the total number of team shots. \\
\hline Productivity & Number of points scored per 10 possessions. \\
\hline Average attack / score & Mean number of attacks required to score. \\
\hline Defensive efficiency & Number of opposition attacks which do not result in a shot as a percentage of their total attacks. \\
\hline Defensive actions & Number of fouls, turnovers and tackles combined per pitch zone. \\
\hline
\end{tabular}

(Table 1) were then devised and referenced during event tagging to ensure consistency 
and accuracy of coding (Allister et al., 2018; Bradley \& O'Donoghue, 2011; James, Mellalieu, \& Jones, 2005).

\subsection{Reliability assessment}

To determine intra-rater reliability, 2 games were randomly selected and coded twice over a 4-week period. Using the convention outlined by McGraw and Wong (1996), a two-way mixed effects model, evaluating absolute agreement between the mean of 4 full-game measurements, was selected to compute the intraclass correlation coefficient (ICC). The lowest ICC recorded was 0.93 (the number of unsuccessful hand passes), all other variables had an ICC >0.93 (mean 0.98), demonstrating excellent reliability (Koo \& Li, 2016).

\subsection{Statistical analysis}

The relative difference between winners and losers was analysed using SPSS for Windows (Version 24; SPSS Inc., Chicago, USA) with statistical significance accepted at $p \leq 0.05$. Descriptive statistics are presented as mean \pm SD. Game statistics and performance indicators were evaluated to provide a preliminary univariate analysis. Differences between winners and losers that were found to be normally distributed using the Shapiro-Wilk test were analysed using a one-sample t-test. Differences which did not reflect a normal distribution, were examined using a Wilcoxan signed rank test. Prior to the PCA being employed, preliminary screening excluded 48 variables due to observed functional dependencies and distributional range. A correlation matrix was subsequently used to identify and provisionally remove any of the 35 remaining variables that were highly correlated with others. The PCA was then conducted on the differences between winners and losers using an orthogonal rotation (Varimax with Kaiser normalisation). Previously excluded variables were then progressively 
incorporated into the PCA, using a trial and error approach, to optimise the KaiserMeyer-Olkin (KMO) measure of sampling adequacy. The KMO statistic of 0.73 achieved was deemed sufficient and all individual KMO values were above the acceptable limit of 0.5 (Kaiser \& Rice, 1974). Overall, 18 variables were retained with an average communality of 0.82 (range $0.58-0.94$ ). The PCA produced 4 components with eigenvalues greater than Kaiser's criterion of 1 (Kaiser, 1970). The associated regression factors were then evaluated using discriminate analysis (SPSS) and logistic regression (RStudio Team 2015; Integrated Development for R. RStudio, Inc., Boston, USA), with both models incorporating a leave-one-out cross-validation (LOOC) to compare how well these techniques correctly classified winners and losers.

\section{Results}

\subsection{Match characteristics}

The average (mean \pm SD) playing and ball in play times were 74:12 $\pm 1: 38$ and 37:08 \pm 3:25 min:sec, respectively. There were no significant differences demonstrated by winners in the Elo ratings (1822.1 \pm 184.6 vs. $1753.6 \pm 174.6)$, number of substitutions made $(5.0 \pm 1.2$ vs. $5.5 \pm 0.6)$, or black $(0.4 \pm 0.6$ vs. $0.6 \pm 0.7)$, yellow $(1.7 \pm 1.4$ vs $1.2 \pm 1.1)$ or red $(0.2 \pm 0.4$ vs. $0.2 \pm 0.4)$ cards received, compared to losers. The main insights from the results are summarised below according to aspects of game play (Tables 2-4) and pitch location origin (Table 5).

\subsection{Univariate analysis}

Shots, shot efficiency, total score, total number of scores, average attack per score, productivity, points, points from play, and goals of winners was significantly superior to losers (Table 2). When compared to losing teams, winning teams had a significantly 
higher number of turnovers (Table 3). However, there were no significant differences in tackles, defensive actions or defensive efficiency between winners and losers. There were no significant differences in any indices of either team or individual possession (Table 3). Winners had a significantly higher percentage of hand pass success and a significantly lower percentage of unsuccessful hand passes than losers (Table 4). Winners also performed significantly less kick outs, resulting in fewer successful kick outs and successful dead ball kick passes overall. There were no significant differences between winners and losers in selected variables examined across pitch locations (Table $5)$.

Table 2. Univariate differences in offensive play, mean \pm SD.

\begin{tabular}{lccccc}
\hline & \multicolumn{2}{c}{ Offensive play } \\
\hline Variable & Winners & Losers & Variable & Winners & Losers \\
\hline Attack (n) & $41.4 \pm 6.6$ & $38.4 \pm 5.7$ & Total score & $18.1 \pm 4.8^{\beta}$ & $13.0 \pm 4.2$ \\
Attack efficiency (\%) & $71.6 \pm 10.3$ & $68.3 \pm 8.0$ & Total no. scores (n) & $15.5 \pm 3.5^{\alpha}$ & $11.9 \pm 3.1$ \\
Shot (n) & $29.5 \pm 5.7^{\alpha}$ & $26.2 \pm 4.8$ & Av. attack / score (n) & $2.9 \pm 1.0^{\alpha}$ & $3.5 \pm 1.3$ \\
Shot from play (n) & $22.8 \pm 6.4$ & $19.7 \pm 5.0$ & Productivity & $2.5 \pm 0.7^{\alpha}$ & $1.8 \pm 0.6$ \\
Shot from play (\%) & $76.1 \pm 9.9$ & $74.8 \pm 8.3$ & Point (n) & $14.1 \pm 3.1^{\alpha}$ & $11.3 \pm 2.9$ \\
Shot from dead ball (n) & $6.8 \pm 2.1$ & $6.5 \pm 2.0$ & Point from play (n) & $9.5 \pm 3.1^{\alpha}$ & $7.3 \pm 2.6$ \\
Shot from dead ball (\%) & $23.9 \pm 9.9$ & $25.2 \pm 8.3$ & Point from dead ball (n) & $4.6 \pm 2.0$ & $4.0 \pm 2.3$ \\
Shot efficiency (\%) & $53.2 \pm 11.5^{\alpha}$ & $45.6 \pm 9.5$ & Goal (n) & $1.3 \pm 1.0^{\beta}$ & $0.6 \pm 0.9$ \\
\hline
\end{tabular}

No. = number, Av. = average. Symbols indicate significantly different $(p \leq 0.05)$ from losers, using either a one sample t-test $(\alpha)$ or Wilcoxon signed rank test $(\beta)$.

Table 3. Univariate differences in defensive play and possession, mean $\pm \mathrm{SD}$.

\begin{tabular}{lcclcc}
\hline \multicolumn{2}{c}{ Defensive play } & & \multicolumn{2}{c}{ Possession } \\
\hline Variable & Winners & Losers & Variable & Winners & Losers \\
\hline Turnover (n) & $32.5 \pm 7.5^{\alpha}$ & $28.7 \pm 6.5$ & Team possession (n) & $71.6 \pm 7.7$ & $71.8 \pm 8.6$ \\
Tackle (n) & $90.9 \pm 23.1$ & $92.9 \pm 16.3$ & Team possession (\%) & $51.1 \pm 4.2$ & $48.9 \pm 4.2$ \\
Tackle successful (n) & $10.0 \pm 3.7$ & $9.8 \pm 3.5$ & Team possession (s) & $973.9 \pm 110.2$ & $936.2 \pm 140.5$ \\
Tackle successful (\%) & $10.9 \pm 3.1$ & $10.7 \pm 3.9$ & Team possession av.(s) & $13.8 \pm 2.2$ & $13.3 \pm 3.0$ \\
Tackle unsuccessful (n) & $80.8 \pm 20.7$ & $83.1 \pm 16.1$ & & $298.8 \pm 36.6$ & $297.3 \pm 38.6$ \\
Tackle unsuccessful (\%) & $89.1 \pm 3.1$ & $89.3 \pm 3.9$ & Player possession (n) & $636.2 \pm 92.2$ & $608.6 \pm 115.4$ \\
Free kick won (n) & $19.4 \pm 5.7$ & $18.7 \pm 7.1$ & Player possession (s) & $2.1 \pm 0.2$ & $2.0 \pm 0.2$ \\
Defensive actions* (n) & $142.2 \pm 27.8$ & $141.4 \pm 22.3$ & Player possession av.(s) & & \\
Defensive efficiency (\%) & $31.8 \pm 8.0$ & $28.4 \pm 10.3$ & & \\
\hline Av & & &
\end{tabular}

Av. = average. *Defensive actions include; combined turnovers, tackles and fouls committed (free kick conceded). Symbols indicate significantly different $(p \leq 0.05)$ from losers, using either a one sample t-test $(\alpha)$ or Wilcoxon signed rank test $(\beta)$.

Table 4. Univariate differences in passing and dead ball distribution, mean \pm SD. 


\begin{tabular}{|c|c|c|c|c|c|}
\hline \multicolumn{3}{|c|}{ Passing } & \multicolumn{3}{|c|}{ Dead ball } \\
\hline Variable & Winners & Losers & Variable & Winners & Losers \\
\hline Hand+kick pass (n) & $247.7 \pm 37.1$ & $248.6 \pm 39.2$ & Dead ball (n) & $42.7 \pm 7.4$ & $46.8 \pm 7.3$ \\
\hline HPKP successful (n) & $227.0 \pm 38.5$ & $226.5 \pm 41.2$ & DBKP successful (n) & $28.0 \pm 4.6^{\alpha}$ & $31.1 \pm 5.9$ \\
\hline HPKP successful (\%) & $91.4 \pm 3.1$ & $90.8 \pm 3.3$ & DBKP successful (\%) & $78.8 \pm 8.1$ & $77.2 \pm 8.0$ \\
\hline HPKP unsuccessful (n) & $20.6 \pm 6.2$ & $22.1 \pm 6.2$ & DBKP unsuccessful (n) & $7.9 \pm 3.7$ & $9.3 \pm 3.8$ \\
\hline HPKP unsuccessful (\%) & $8.6 \pm 3.1$ & $9.2 \pm 3.3$ & DBKP unsuccessful (\%) & $21.2 \pm 8.1$ & $22.8 \pm 8.0$ \\
\hline Hand pass (n) & $168.6 \pm 36.6$ & $170.2 \pm 42.0$ & DB free kick pass (n) & $13.5 \pm 4.6$ & $13.5 \pm 6.3$ \\
\hline HP successful (n) & $164.6 \pm 35.8$ & $165.3 \pm 42.3$ & DBFKP successful (n) & $12.7 \pm 4.2$ & $12.7 \pm 5.4$ \\
\hline HP successful (\%) & $97.6 \pm 1.2^{\alpha}$ & $96.9 \pm 1.7$ & DBFKP successful (\%) & $94.4 \pm 5.5$ & $95.5 \pm 6.2$ \\
\hline HP unsuccessful (n) & $4.0 \pm 2.0^{\alpha}$ & $4.9 \pm 2.0$ & DBFKP unsuccessful (n) & $0.8 \pm 0.8$ & $0.8 \pm 1.2$ \\
\hline HP unsuccessful (\%) & $2.4 \pm 1.2^{\alpha}$ & $3.1 \pm 1.7$ & DBFKP unsuccessful (\%) & $5.6 \pm 5.5$ & $4.5 \pm 6.2$ \\
\hline Kick pass (n) & $79.0 \pm 14.0$ & $78.4 \pm 13.7$ & DB kick out (n) & $20.3 \pm 4.2^{\alpha}$ & $24.0 \pm 3.9$ \\
\hline KP successful (n) & $62.4 \pm 13.9$ & $61.2 \pm 11.2$ & DBKO successful (n) & $13.4 \pm 3.2^{\alpha}$ & $16.0 \pm 4.0$ \\
\hline KP successful (\%) & $78.7 \pm 7.0$ & $78.2 \pm 6.0$ & DBKO successful (\%) & $67.3 \pm 14.4$ & $66.2 \pm 12.6$ \\
\hline KP unsuccessful (n) & $16.6 \pm 5.9$ & $17.2 \pm 6.2$ & DBKO unsuccessful (n) & $6.9 \pm 3.5$ & $8.1 \pm 3.2$ \\
\hline KP unsuccessful (\%) & $21.3 \pm 7.0$ & $21.8 \pm 6.0$ & DBKO unsuccessful (\%) & $32.7 \pm 14.4$ & $33.8 \pm 12.6$ \\
\hline
\end{tabular}

HPKP = combined hand pass and kick pass, $\mathrm{HP}=$ hand pass, $\mathrm{KP}=$ kick pass, DBKP = dead ball kick pass, DBFKP $=$ dead ball free kick pass (excludes shots), DBKO = dead ball kick out. Symbols indicate significantly different $(p \leq 0.05)$ from losers, using either a one sample t-test $(\alpha)$ or Wilcoxon signed rank test $(\beta)$.

Table 5. Univariate differences in selected variables by pitch location origin, mean $\pm \mathrm{SD}$.

\begin{tabular}{lcccccc}
\hline \multirow{2}{*}{ Variable } & \multicolumn{2}{c}{ Defence } & \multicolumn{2}{c}{ Midfield } & \multicolumn{2}{c}{ Attack } \\
\cline { 2 - 7 } & Winners & Losers & Winners & Losers & Winners & Losers \\
\hline Attack (n) & $21.6 \pm 5.0$ & $20.4 \pm 5.8$ & $18.0 \pm 5.0$ & $16.6 \pm 4.8$ & $1.8 \pm 1.4$ & $1.4 \pm 1.4$ \\
& & & & & & \\
Turnover (n) & $17.6 \pm 3.5$ & $16.1 \pm 5.1$ & $13.1 \pm 5.4$ & $11.3 \pm 4.8$ & $1.8 \pm 1.5$ & $1.3 \pm 1.3$ \\
Tackle (n) & $37.3 \pm 14.0$ & $39.4 \pm 8.9$ & $41.7 \pm 16.8$ & $39.4 \pm 10.9$ & $11.9 \pm 7.1$ & $14.1 \pm 6.6$ \\
Free kick (n) & $4.9 \pm 2.2$ & $4.0 \pm 2.6$ & $9.0 \pm 3.7$ & $9.7 \pm 5.2$ & $5.4 \pm 2.0$ & $5.0 \pm 2.6$ \\
Defensive actions (n) & $60.0 \pm 15.9$ & $61.0 \pm 12.7$ & $64.5 \pm 20.8$ & $60.2 \pm 15.9$ & $17.7 \pm 9.9$ & $20.2 \pm 8.0$ \\
& & & & & & \\
Team possession (n) & $40.4 \pm 6.4$ & $42.2 \pm 6.7$ & $22.9 \pm 5.4$ & $21.8 \pm 7.1$ & $8.3 \pm 2.7$ & $7.8 \pm 2.6$ \\
Player possession (n) & $82.4 \pm 15.8$ & $84.7 \pm 21.9$ & $146.7 \pm 30.4$ & $144.2 \pm 34.5$ & $70.6 \pm 14.3$ & $68.4 \pm 19.5$ \\
\hline
\end{tabular}

\subsection{Exploratory multivariate analysis}

The 4 eigenvectors produced by the PCA explained $81.9 \%$ of the total variance and the component loadings after rotation are illustrated in Table 6. The dominant variable(s) in each component were used to subjectively characterise new variables reflecting 1) midfield counterattacking, 2) defensive free kick efficiency, 3) defensive counterattacking, and 4) possession. Logistic regression revealed that defensive counterattacking $(\beta$-coefficient $=-3.22, \mathrm{SE}=1.36, p=0.018)$ significantly contributed to outcome (lose vs. win) and was retained in the model (odds ratio; 0.0398, 0.0012- 
$0.2980 ; 95 \% \mathrm{CI}$ ), with the $\log$ likelihood function being $8.00, \chi^{2}(1)=16.00, p<0.001$.

Using lose as positive, the area under the curve was 0.88. Discriminant analysis performed on this variable, revealed 1 discriminant function (win or lose), which explained $100 \%$ of the variance, canonical $\mathrm{R}^{2}=0.47$, significantly differentiating the groups, $\Lambda=0.53, \chi^{2}(1)=13.77, p<0.001$. The LOOC returned and accuracy of $87.5 \%$ in both models.

Table 6. Summary of exploratory PCA using 18 variables created from the differences between independent winners ( $\mathrm{n}=12)$ and losers $(\mathrm{n}=12)$.

\begin{tabular}{|c|c|c|c|c|}
\hline \multirow[b]{2}{*}{ Principal component } & \multicolumn{4}{|c|}{ Rotated component loadings } \\
\hline & $\begin{array}{c}\text { Midfield } \\
\text { counterattacking }\end{array}$ & $\begin{array}{l}\text { Defensive free } \\
\text { kick efficiency }\end{array}$ & $\begin{array}{c}\text { Defensive } \\
\text { counterattacking }\end{array}$ & Possession \\
\hline \multicolumn{5}{|l|}{ Variable } \\
\hline dAttack origin MF & 0.958 & 0.123 & 0.060 & -0.014 \\
\hline dTeam possession origin MF & 0.913 & 0.320 & 0.031 & 0.077 \\
\hline dTurnover origin $\mathrm{MF}$ & 0.880 & -0.136 & 0.353 & -0.130 \\
\hline dPlayer possession origin AT & 0.608 & -0.639 & 0.209 & 0.110 \\
\hline dTackle origin AT & 0.458 & -0.740 & -0.133 & -0.325 \\
\hline dShots from play & 0.664 & -0.315 & 0.589 & 0.135 \\
\hline dPoints from play & 0.309 & 0.074 & 0.686 & -0.138 \\
\hline dAttack origin DF & -0.155 & -0.544 & 0.618 & -0.362 \\
\hline dTurnover origin DF & -0.561 & 0.117 & 0.601 & 0.452 \\
\hline dPlayer possession time & 0.017 & 0.091 & 0.404 & 0.824 \\
\hline dDead ball free kick pass unsuccessful & 0.156 & 0.551 & -0.192 & 0.518 \\
\hline dDead ball free kick pass successful & 0.081 & 0.873 & -0.104 & 0.126 \\
\hline dFree kick origin DF & -0.353 & 0.759 & 0.136 & 0.208 \\
\hline dPlayer possession origin DF & -0.759 & 0.425 & 0.008 & 0.325 \\
\hline dTackle origin MF & 0.165 & -0.307 & 0.235 & -0.739 \\
\hline dDead ball kick out successful & -0.159 & 0.048 & -0.893 & -0.161 \\
\hline dTackle origin DF & -0.694 & 0.305 & 0.039 & -0.083 \\
\hline dTeam possession origin DF & -0.833 & 0.383 & -0.259 & 0.157 \\
\hline Eigenvalue & 5.94 & 3.67 & 2.90 & 2.23 \\
\hline$\%$ of variance & 33.01 & 20.41 & 16.12 & 12.38 \\
\hline
\end{tabular}

\section{Discussion}

This is the first investigation in Gaelic football to use PCA on an extensive range of existing variables to establish a novel performance indicator, defensive 
counterattacking, which effectively differentiated winners from losers with a classification accuracy of $87.5 \%$. This was superior to the $71 \%$ reported previously using discriminate analysis in Gaelic football (Allister et al., 2018) and comparable to the $88 \%$ revealed using logistic regression in Australian football (Robertson et al., 2016). Univariate analysis indicated that winners had a significantly higher total score and number of scores, reflected in more shots, points, goals and points from play being achieved. Winners were characterised by having a significantly better; shot efficiency, average attack per score, and productivity (scores per 10 possessions). Compared to losers, winners gained significantly more turnovers and completed a higher percentage of successful hand passes and consequently a lower percentage of unsuccessful hand passes. Winners executed significantly less dead ball kick passes and kick outs, however their success rate was comparable to losers.

In addition to outscoring the opposition, it is likely that successful Gaelic football teams regain possession through turnovers; generated via tackling, interceptions and/or unforced technical errors. In the present study, winners had significantly more turnovers than losers confirming previous results (Carroll, 2013). Both winners and losers generated $\sim 55 \%$ of their turnovers in defence and $\sim 40 \%$ in midfield. Turnovers are often produced from organised tackling strategies, although presently only $\sim 11 \%$ of tackles performed by winners and losers were deemed successful, perhaps due to poor technical execution and/or a focus of tackling the player instead of the ball. The highest numbers of tackles in both winners and losers were recorded in midfield, reflecting the congested nature of this highly contested zone. In accordance with previous foul distribution results (Mangan et al., 2017), both winners and losers committed $\sim 50 \%$ of their fouls in midfield, suggesting that teams may have employed a defensive press in this zone. 
Currently, when not in possession, teams withdraw all or some of their attacking players to establish a defensive zone, ranging from 45 or $65 \mathrm{~m}$ from their goal line, within which defensive actions are concerted. This concentrated defensive tactic can result in turnovers if attacking teams do not have the tactical ability to penetrate or technical skill to shoot for scores from long range. Although these defensive formations are often viewed negatively within the media, it is likely that this tactic contributed to the higher number of turnovers acquired by both winners and losers in defence and midfield. Overall, winners performed more combined defensive actions (turnovers, tackles and fouls committed) in midfield, whereas losers committed slightly more in defence, supporting previous results (Mangan et al., 2017). Between the 1980s and 2014-16, there has been an increase from 51 to $56 \%$ in the percentage of passes completed in midfield (Lynch \& Carroll, 2017) as teams retain possession and patiently try to engineer attacks through organised formations or invite the opposition to flex their defensive positions. Therefore, a high press strategy may produce more turnovers in midfield and attack enabling counterattacks to be instigated in closer proximity to the opposition's goal, and may even occur before the opposing team have had time to organise their defensive system.

The midfield and defensive counterattacking variables identified (Table 6), highlight the importance of gaining turnovers and instigating counterattacks that enable attacking players to have opportunities to shoot (and score) from play. While the outcome of turnovers originating in defence or midfield were not examined, the importance of defensive counterattacking in differentiating between winners and losers was clearly evident and supports previous findings (Bradley \& O’Donoghue, 2011). Although defensive turnovers require the ball to be transferred, often through a large number of players towards the opposition's goal, they can however result in successful 
counterattacks as opposition players are often committed to attack. Even though successful teams are cognisant of this risk and retain a degree of defensive structure, counterattacks incorporating intelligent deployment of offensive players and successful execution of technical skills enable penetration of both organised and disorganised defensive systems.

Similar to successful club teams (McGuigan et al., 2018), the attacking strategy of winning inter-county teams in the present study was associated with a more favourable average attack to score and productivity rating compared to losers. The number of team possessions in both winners and losers were similar to those reported previously (Mangan et al., 2017) with 4 out of every 10 possessions resulting in a scoring attempt. Three quarters of the shots were taken from open play, and the importance of this performance indicator was reflected in its inclusion in both the midfield- and defensive- counterattacking components produced by the PCA. In this study of NFL and AIC games, shot efficiency differentiated winners (53\%) from losers (46\%), replicating previous findings involving AIC games (Allister et al., 2018; Carroll, 2013), and suggests enhanced technical performance or decision making ability of winners (McGahan et al., 2018). The 97\% success rate for hand passes was identical to that recently reported (Lynch \& Carroll, 2017; Mangan et al., 2017) and reinforces the importance placed by coaches on this mode of ball transfer. The high hand pass retention rate is likely to have contributed to the effective attacks previously highlighted.

The importance of dead balls in influencing match outcome is evident by the fact that $\sim 60 \%$ and $65 \%$ of team possessions for winners and losers, respectively are instigated from a restart. In winners, kick outs accounted for $48 \%$ of dead balls, in 
comparison to the $51 \%$ reported for losers. The average kick out success achieved by winners and losers $(\sim 66.5 \%)$ across full games was higher than the results reported previously for top $(61 \%)$ and bottom $(56 \%)$ teams (Carroll, 2013) and may be attributed to improved coaching, technical ability, contemporary strategies directed towards ball retention (e.g. short kick outs) and/or opposition tactics (i.e. employing a high press or deep defensive shield). Both kick outs and defensive free kicks provide a platform for teams to execute their offensive strategies. Not surprisingly, $95 \%$ of all passes from free kicks were retained by both winners and losers, outlining the primary emphasis on ball retention in developing an attack.

The methodological limitations should be considered in interpreting the findings of this study. Although the match sample included semi-finals and finals from both the NFL and AIC, the performance profiles may have been diminished by results obtained from early stages of the NFL. The sample size precluded a comparison of NFL and AIC games. A detailed literature review identified the performance indicators examined, however, it is possible that other important variables were excluded. Derived variables such as global defensive actions could be refined to exclude nonintentional (i.e. technical) fouls. Future research should investigate differences in the technical, tactical and physical components of winning teams across halves and quarters and evaluate whether temporal decrements in performance occur. Furthermore, examination of the technical profiles of successful players would enable position specific benchmarks to be developed.

\section{Conclusion}

This exploratory investigation has highlighted the potential of using PCA to combine a large number of existing performance indicators into new aggregated variables. Using 
the novel defensive counterattacking component, winners were differentiated from losers with an accuracy of $87.5 \%$. The combined use of univariate and multiple multivariate analyses addresses some of the limitations of previous studies conducted in Gaelic football and enhances the current literature base. The findings confirm the importance of developing tactical strategies that enable turnovers in the defensive area to be converted into counterattacks, and supports the subjective evaluation often highlighted and promoted by coaches. The performance indicators and winning profiles presented can be used as a reference for coaches, support staff and researchers to inform current preparation programmes and possible future studies.

\section{Acknowledgements}

The authors would like to thank the players and teams who participated in the study.

\section{Disclosure statement}

No potential conflict of interest was reported by the authors.

\section{Funding}

No external sources of funding were provided for this research 


\section{References}

Allister, A., Byrne, P. J., Nulty, C. D., \& Jordan, S. (2018). Game-related statistics which discriminate elite senior Gaelic football teams according to game outcome and final score difference. International Journal of Performance Analysis, 18(4), $622-632$.

Beasley, K. J. (2015). Nutrition and Gaelic football: Review, recommendations, and future considerations. International Journal of Sport Nutrition and Exercise Metabolism, 25(1), 1-13.

Bradley, J., \& O’Donoghue, P. (2011). Counterattacks in elite Gaelic football competition. International Journal of Performance Analysis in Sport, 11(1), $159-170$.

Carroll, R. (2013). Team performance indicators in Gaelic football and opposition effects. International Journal of Performance Analysis in Sport, 13(3), 703-715.

Castellano, J., Casamichana, D., \& Lago, C. (2012). The use of match statistics that discriminate between successful and unsuccessful soccer teams. Journal of Human Kinetics, 31(1), 137-147.

Field, A. (2018). Discovering Statistics Using IBM SPSS Statistics (5 ${ }^{\text {th }}$ Edition). London: Sage.

Gamble, D., Spencer, M., McCarren, A., \& Moyna, N. (2019). Activity profile, PlayerLoad ${ }^{\mathrm{TM}}$ and heart rate response of Gaelic football players: A pilot study. Journal of Human Sport and Exercise, 14(4), 1-15.

Hastie, T., Tibshirani, R., \& Friedman, J. (2009). The Elements of Statistical Learning - Data Mining, Inference, and Prediction, Second Edition. New York: Springer.

Hughes, M. D., \& Bartlett, R. M. (2002). The use of performance indicators in performance analysis. Journal of Sports Sciences, 20, 739-754.

James, N., Mellalieu, S. D., \& Jones, N. M. P. (2005). The development of positionspecific performance indicators in professional rugby union. Journal of Sports Sciences, 23(1), 63-72.

Jones, N. M. P. P., Mellalieu, S. D., \& James, N. (2004). Team performance indicators as a function of winning and losing in rugby union. International Journal of Performance Analysis in Sport, 4(1), 61-71.

Kaiser, H. F. (1970). A second generation little Jiffy. Psychometrika, 35(4), 401-415.

Kaiser, H. F., \& Rice, J. (1974). Jiffy, Little Mark Iv,. Educational and Psychological Measurement, 34, 111-117.

Koo, T. K., \& Li, M. Y. (2016). A guideline of selecting and reporting intraclass correlation coefficients for reliability research. Journal of Chiropractic Medicine, 15(2), 155-163. 
Lynch, P., \& Carroll, R. (2017). To compare the type of passing in Gaelic football at senior inter-county level historically to modern day. International Journal of Performance Analysis in Sport, 17(6), 1-10.

Malone, S., Solan, B., \& Collins, K. (2017). The running performance profile of elite Gaelic football match-play. Journal of Strength and Conditioning Research, 31(1), 30-36.

Mangan, S., \& Collins, K. (2016). A rating system for Gaelic football teams: Factors that influence success. International Journal of Computer Science in Sport, 15(2), 78-90.

Mangan, S., Ryan, M., Devenney, S., Shovlin, A., McGahan, J., Malone, S., ... Collins, K. (2017). The relationship between technical performance indicators and running performance in elite Gaelic football. International Journal of Performance Analysis in Sport, 17(5), 706-720.

McGahan, J. H., Mangan, S., Collins, K., Burns, C., Gabbett, T., \& Neill, C. O. (2018). Match-play running demands and technical performance among elite Gaelic footballers: Does divisional status count? Journal of Strength and Conditioning Research, DOI: 10.1519/JSC.0000000000002450.

McGraw, K. O., \& Wong, S. P. (1996). Forming inferences about some intraclass correlation coefficients. Psychological Methods, 1(1), 30-46.

McGuigan, K., Hughes, M., \& Martin, D. (2018). Performance indicators in club level Gaelic football. International Journal of Performance Analysis in Sport, 18(5), 780-795.

O’Donoghue, P. (2009). Research methods for sports performance analysis. London: Routledge.

Ofoghi, B., Zeleznikow, J., MacMahon, C., \& Raab, M. (2013). Data mining in elite sports: A review and a framework. Measurement in Physical Education and Exercise Science, Vol. 17, pp. 171-186.

Reilly, T., \& Collins, K. (2008). Science and the Gaelic sports: Gaelic football and hurling. European Journal of Sport Science, 8(5), 231-240.

Robertson, S., Back, N., \& Bartlett, J. D. (2016). Explaining match outcome in elite Australian Rules football using team performance indicators. Journal of Sports Sciences, 34(7), 637-644.

Rooney, L., \& Kennedy, R. (2018). Home advantage in Gaelic football : the effect of divisional status, season and team. International Journal of Performance Analysis in Sport, 18(6), 917-925.

RStudio Team 2015. Integrated Development for R. Boston, U.S.A: RStudio, Inc.

Watson, N., Durbach, I., Hendricks, S., \& Stewart, T. (2017). On the validity of team performance indicators in rugby union. International Journal of Performance Analysis in Sport, 17(4), 609-621. 
